\title{
Implantación de prótesis aórtica transcatéter (TAVI) por vía Femoral. Estado actual (2015). Visión del Cardiólogo intervencionista
}

\author{
Transfemoral transcatheter aortic valve implantation (TAVI). \\ Current state of affairs (2015). The interventional cardiologist view.
}

\author{
Nicolás Vázquez González, Jorge Salgado Fernández, Ramón Calviño Santos \\ Unidad de Hemodinámica y Cardiología Intervencionista. Servicio de Cardiología. Complexo Hospitalario Universitario A Coruña (CHUAC).
}

\section{Introducción}

Estamos asistiendo a una de las mayores revoluciones que ha experimentado la cardiología en muchas décadas: el inicio del implante valvular aórtico transcatéter (TAVI) para el tratamiento de la estenosis valvular aórtica. Es esta una tecnología disruptiva. Se definen como tales, a aquellas tecnologías o innovaciones que conducen a la aparición de productos, que compiten con una tecnología dominante para cambiar el "status quo" en el mercado o también: "Aquella que, de forma inesperada, desplaza a otra ya existente". Este es exactamente el contexto en el que se mueve esta técnica.

Desde 2002, se ha tratado con TAVI a más de 150.000 pacientes en todo el mundo y se espera que la cifra crezca, de forma importante, debido al incremento de la esperanza de vida de la población, que producirá un gran aumento de los pacientes con estenosis aórtica senil. Además, los buenos resultados generan una tendencia al tratamiento de otras situaciones, como las prótesis aórticas quirúrgicas degeneradas e incluso insuficiencias aórticas. La primera prótesis transcatéter se implantó el 16 de abril de 2002 en Rouen por el Dr. Alain Cribier en un paciente de 57 años con múltiples comorbilidades y por tanto es un hito en la historia de la cardiología.

A pesar de los excelentes resultados, todavía existen limitaciones y problemas, que mejoran de forma continua debido que las compañías desarrollan sistemas cada vez de menor perfil y mejores características y nuevos prototipos, con el fin de disminuir estas limitaciones.

El objetivo de esta revisión es la puesta al día del conocimiento del procedimiento, los resultados, limitaciones y las perspectivas de futuro de una tecnología que está cambiando la historia de la Medicina en general y de la Cardiología en particular

\section{Dimensión del problema}

La estenosis aórtica degenerativa es la valvulopatía más frecuente y su prevalencia está aumentando como consecuencia del incremento de la esperanza de vida. Si conti- núa el grave problema demográfico, con el aplanamiento de la pirámide poblacional, en el año 2050 seremos el segundo país más viejo, detrás de Japón.

En España, un 7\% de los mayores de 85 años tienen estenosis aórtica severa. Según datos del Instituto Nacional de Estadística, hay 1,5 millones de habitantes de más de 85 años de edad, por lo que se estima que 100.000 van a sufrir la enfermedad.

Cuando aparecen los síntomas, la enfermedad progresa rápidamente y se ensombrece el pronóstico. La mortalidad de los pacientes asintomáticos es del 2,3\% por año y la muerte súbita es todavía menos frecuente pero, se debe considerar la cirugía o la TAVI cuando la estenosis es grave y el paciente está sintomático. En los pacientes ancianos puede ser difícil interpretar los síntomas como la disnea 0 el sincope, y además, no es infrecuente la presencia de enfermedad coronaria cuando el paciente tiene angina (más del $50 \%$ de los pacientes con estenosis aórtica tienen enfermedad coronaria). La realización de una ergometría puede contribuir a aclarar esta situación, ya que un síncope, una disnea importante, la falta de incremento 0 caída tensional con el ejercicio, pueden hacer que un paciente considerado como asintomático pase a serlo, sobre todo, teniendo en cuenta que estos pacientes pueden ir autolimitandose de forma progresiva e inconsciente.

Existe acuerdo general en que cuando la estenosis aórtica es grave y está muy calcificada, la supervivencia a los 2 años de seguimiento no es superior al $50 \%$. Esto hace que los pacientes asintomáticos deban de ser evaluados cada 6-12 meses y cuando aparecen síntomas considerar el recambio valvular o la TAVI.

\section{Tipos de válvulas}

Existen muchos dispositivos en el mercado en distintas situaciones de uso, pero los más implantados, con mucho, son la válvula Edwards Sapien y la CoreValve Medtronic que acumulan toda la evidencia científica y son las que describiremos con más detenimiento (Figura 1). 
Figura 1. Principales prótesis aórticas transcatéter

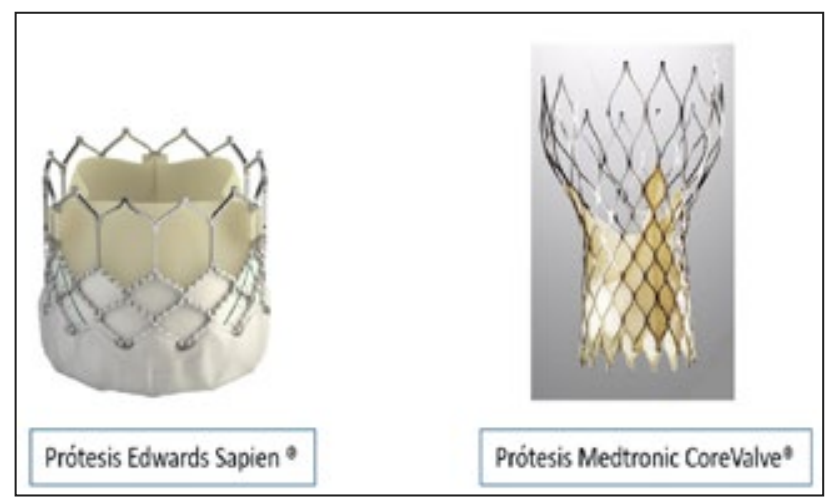

Válvula Edwards: es el desarrollo de la primera de Cribier,

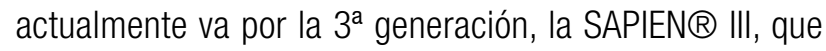
aporta muchas mejoras respecto al anterior. Se trata de una válvula de pericardio bovino montada en un Stent de cromo cobalto expandible con balón y un sistema de liberación del $14 \mathrm{~F}$ que minimiza el trauma vascular y permite un posicionamiento coaxial que facilita el despliegue.

Durante la implantación es preciso realizar sobreestimulación ventricular rápida para evitar su desplazamiento durante el inflado. Desde el 2007 tiene marca CE para el implante transfemoral (TF).

Está disponible en diámetros de 19, 23, 26 y 29 mm que cubren anillos entre 18 y $27 \mathrm{~mm}$.

Válvula CoreValve® (Medtronic Inc.): tiene tres valvas de pericardio porcino suturadas sobre un stent de nitinol autoexpandible. El nitinol es muy moldeable a baja temperatura, lo que facilita su introducción en el catéter de liberación, mientras que con la temperatura corporal recupera su forma. El calibre del sistema liberador es de 18 Fr. La válvula, una vez implantada, se sitúa en posición supranular. Su estructura tiene celdas más amplias a nivel proximal para facilitar el acceso a los ostium coronarios. No se requiere sobreestimulación ventricular rápida y una vez que se han liberado parcialmente ya funciona. Hasta cierto momento puede recolocarse o reposicionarse parcialmente. Disponible en diámetros de 23, 26, 29 y 31 mm que cubren rangos de anillo de 18 a $31 \mathrm{~mm}$.

Aunque ya hay otras prótesis disponibles en el mercado, estas dos representan los dos mecanismos básicos actuales: las prótesis autoexpandibles y las expandibles con balón. En la tabla 1 se muestran las principales prótesis, aunque hay otros muchos prototipos en fase de prueba y en distintos estadíos de avaluación clínica.

\section{Evidencia}

La evidencia científica se fundamenta en varios registros observacionales y tres estudios con distribución aleatoria. En estos estudios se analizan los datos en términos de éxito, complicaciones vasculares, necesitad de marcapasos y mortalidad inmediata, a 30 días y a medio y largo plazo (5 años) y mejoría de la calidad de vida (grado funcional postintervención).
Tabla 1. Principales prótesis, fabricante, calibre y posición en el mercado

\begin{tabular}{|l|l|c|l|l|l|l|}
\hline Prótesis & Fabricante & Calibre (F) & Tipo* & Via** & Tejido & Situación*** \\
\hline Sapien XT & Edwards & $18-19$ & EB & TF-TA-TAo & Bovino & Marca CE \\
\hline Sapien III & Edwards & 14 & EB & TF-TA-TAo & Bovino & Marca CE \\
\hline Centera & Edwards & 14 & AE & TF-TA & Bovino & FIM \\
\hline CoreValve & Medtronic & $18-19$ & AE & TF-TA-TAx & Porcino & Marca CE \\
\hline CV Evolut & Medtronic & 18 & AE & TF & Porcino & Marca CE \\
\hline Engager & Medtronic & 29 & AE & TA-TAo & Bovino & Marca CE TA \\
\hline Sandra Lotus & Boston & 18 & AE & TF & Bovino & Marca CE \\
\hline Direct Flow & Direct flow & 18 & AESS & TF-TAO & Bovino & Marca CE \\
\hline Portico & San Jude & $18-24$ & AE & TF-TA & Bovino & Marca CE TF \\
\hline
\end{tabular}

"EB= empendible con Balón, AEz autoerpandilbe, AESS= anillos expancilbes sin Stent

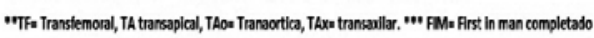

\section{Estudios con distribución aleatoria}

Los estudios fundamentales son los estudios PARTNER (The Placement of Aortic Trancateter Valves) que demuestran, que en los pacientes de alto riesgo quirúrgico o inoperables, se obtienen unas mejorías muy importantes de la supervivencia y los episodios de rehospitalización, en ambos casos muy significativos. Hay que tener en cuenta que en la población normal sin comorbilidades, de igual sexo y edad, la mortalidad esperada a los 5 años es del 40,5\%.

Además, han ofrecido mucha información relacionada, no solo con los resultados de la TAVI, sino también con la historia natural de la estenosis aortica severa. Según datos históricos obtenidos de estudios observacionales en pacientes con estenosis aórtica severa sintomática no sometidos a reemplazo valvular aórtico, se aceptaba universalmente que la supervivencia media de estos pacientes, una vez iniciada la insuficiencia cardiaca era de 2 años. El estudio PARTNER confirma este hecho en un grupo actual, más numeroso y randomizado, en los cuales la supervivencia media es solo de un año. Es de destacar que este es el primer ensayo randomizado, y probablemente el último que se realice en este tipo de pacientes.

Este es un ensayo multicéntrico y randomizado que consta de dos partes diferenciadas:

- PARTNER 1A, que compara la TAVI con el recambio valvular aórtico convencional en pacientes de alto riesgo quirúrgico.

- PARTNER 1B, que compara la TAVI con el tratamiento conservador (podía incluir la Valvuloplastia aórtica).

En ellos se demuestra que en los pacientes de alto riesgo quirúrgico o inoperables se obtienen unas mejorías muy importantes de la supervivencia y los episodios de rehospitalización, en ambos casos muy significativos. También se demostró que en los pacientes de alto riesgo no había diferencias significativas en los resultados entre la TAVI y la cirugía aórtica convencional. 
Recientemente se han publicado los resultados a 5 años de estos estudios, lo que permite un análisis realista con una perspectiva a medio-largo plazo. En el grupo de pacientes con TAVI la mortalidad a 5 años es del 71.8\% (muy alta) comparada con el $93.6 \%$ de los pacientes en el grupo con tratamiento conservador (Figura 2). De los 6 pacientes vivos en el grupo estándar solo uno no sufrió un remplazo aórtico, aunque si una valvuloplastia aórtica durante el seguimiento y de los 5 restantes dos recibieron una TAVI en otro país, 2 fueron sometidos a reemplazo quirúrgico y a uno se le realizo una derivación con conducto valvulado apical a Aorta descendente. La supervivencia media fue de 31 meses en el grupo TAVI contra 11,7 en el grupo conservador $(p<0.0001)$. El riesgo de mortalidad cardiovascular a 5 años es del $57,5 \%$ en TAVI y de 85,9\% en el grupo de tratamiento estándar. La mortalidad no cardiovascular fue del 34\% comparada con el 17\% del grupo estándar, lo que hace hincapié en que las comorbilidades asociadas juegan un importante papel, de ahí la importancia de la selección de candidatos. La incidencia de ACV es del $16 \%$ en el grupo TAVI y del $18,2 \%$ en el grupo estándar.

Figura 2. Mortalidad total (superior) y mortalidad cardiovascular en el PARTNER 1B (inferior)
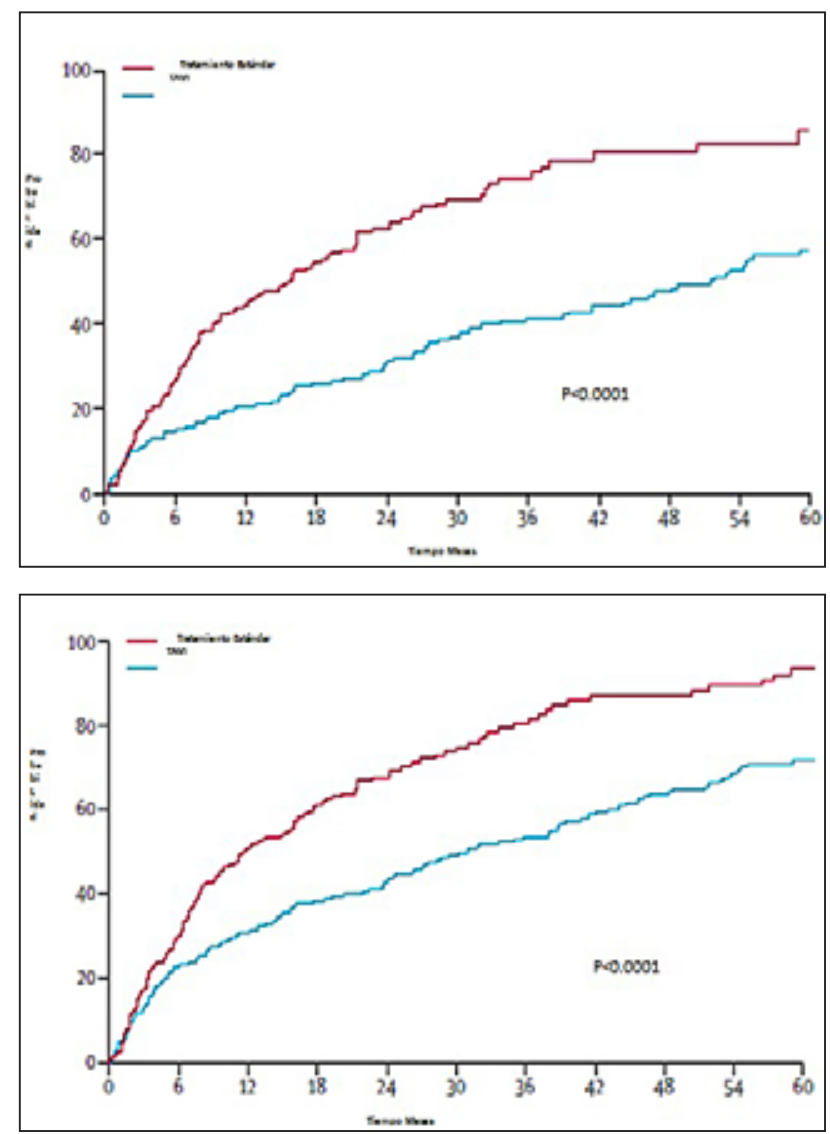

Otra información capital es la relativa a la durabilidad de la válvula ya que en estos pacientes no se objetivaron datos de deterioro estructural o migración y la mejora del área valvular y los gradientes se mantuvieron a los 5 años.

Los resultados a 5 años del PARTNER $1^{\text {a }}$ (Figura 3) muestran una mortalidad (por intención de tratar) del 67,8\% en el grupo TAVI y $62,4 \%$ en el grupo quirúrgico. En caso de abordaje transfemoral la mortalidad es del 63\%. La supervivencia media es de 45,5 meses en el grupo TAVI y 40,6 en el grupo quirúrgico $(p=0,76)$. La incidencia de ACV fue del $14,7 \%$ en TAVI y 15,9 en cirugía. La necesidad de readmisión hospitalaria fue similar en ambos grupos.

Figura 3. Supervivenvia a largo plazo PARTNER 1A

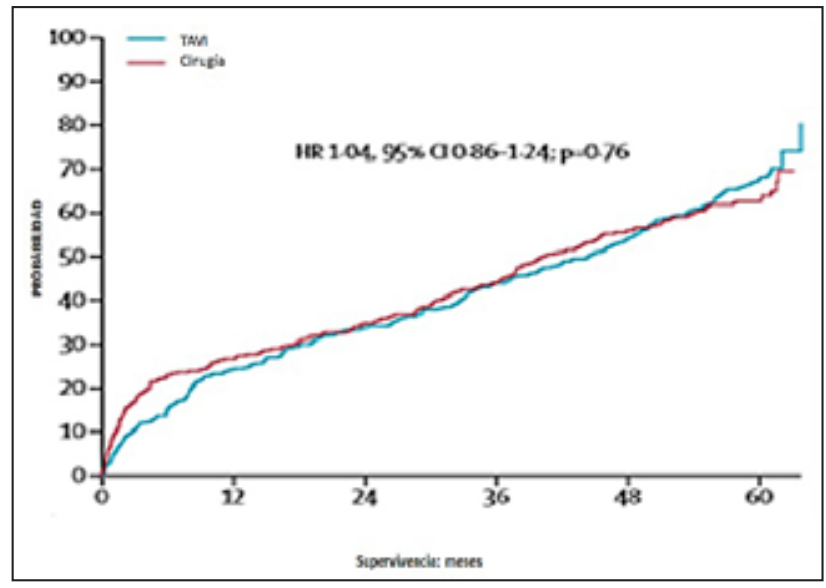

El estado funcional fue también similar con un $85 \%$ (TAVI) y un $81 \%$ (recambio Valvular quirúrgico) respectivamente, de Ios pacientes supervivientes en clase funcional I-II, sin datos de disminución del área valvular, aumento de los gradientes 0 deterioro estructural de la válvula que requiriera recambio. La incidencia de insuficiencia aórtica moderada o severa fue del $14 \%$ en el grupo de TAVI (generalmente paravalvular) y del $1 \%$ en el quirúrgico y tuvo repercusión en la supervivencia ya que la mortalidad a los 5 años fue superior $72,4 \%$ que con insuficiencia moderada o menor $(56,6 \%)$.

Al analizar los resultados de este ensayo se deben de considerar varios factores importantes a tener en cuenta para su análisis en el momento actual.

- En primer lugar, estos ensayos fueron realizados con la $1^{\text {a }}$ generación de dispositivos que tenían un gran calibre y que no representan las grandes mejoras actuales de los mismos.

- En segundo lugar, se realizó en muchos centros en los que era la primera vez que utilizaban este dispositivo, por lo que no se puede descartar la incidencia desfavorable del aprendizaje, sobre todo en la fase inicial, en la tasa de complicaciones vasculares e insuficiencia paravalvular.

- $Y$ en tercer lugar, incluye pacientes de muy alto riesgo con un STS medio del $12 \%$, que es muy superior al de otros estudios de pacientes con alto riesgo 0 inoperables, en que el STS medio era del $7 \%$.

Por tanto, los resultados clínicos y rendimiento de la válvula solo representan los resultados de este dispositivo y no pueden extrapolarse a los resultados esperables con los 
nuevos dispositivos y experiencia actual de los operadores. La selección de pacientes representa la práctica clínica del momento y no la actual.

El tercer estudio randomizado es el Corevalve US Pivotal Trial (M. Reardon. ACC 2015) realizado con la válvula autoexpandible Medtronic CoreValve $®$ que es el único ensayo clínico que ha demostrado la superioridad de un tratamiento transcatéter sobre un tratamiento quirúrgico convencional en términos de mortalidad con una incidencia de ACV similar (Figura 4).

Figura 4. Muerte e incidencia de ACV a dos años en el CoreValve US Pivotal Trial
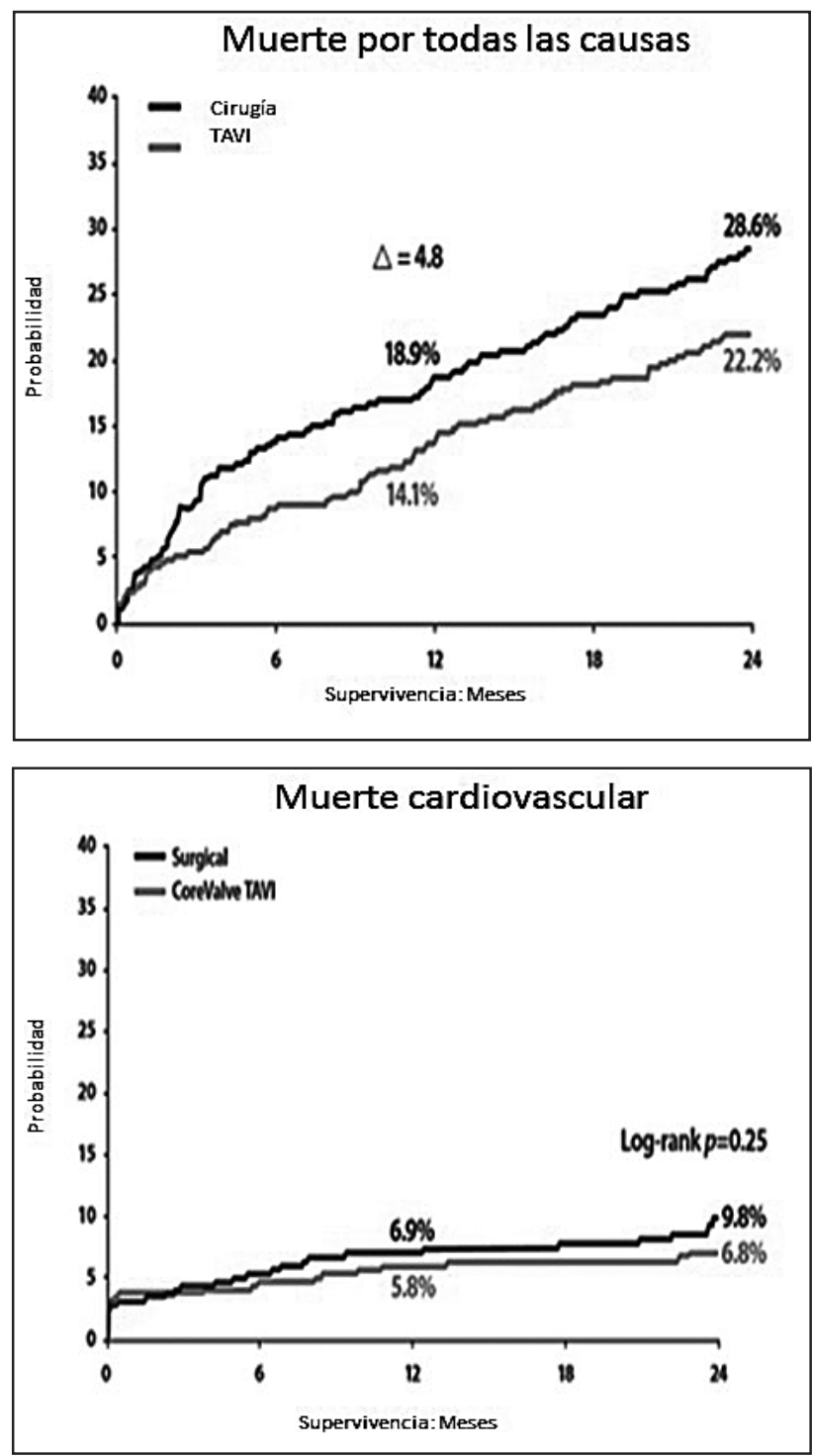

En este ensayo se distribuyó un total de 795 pacientes de elevado riesgo quirúrgico a TAVI o RVAo convencional. La mortalidad al año fue menor con TAVI que con cirugía (el 14,2 frente al 19,1\%). La incidencia de eventos adversos cardiovasculares y ACV al año fue inferior con TAVI.

Este es el primer y único trabajo con distribución aleatoria que demuestra mayor supervivencia después de 2 años en Ios pacientes con TAVI comparados con la cirugía en pacientes de alto riesgo, representa, por tanto, un hito en la historia del TAVI y el tratamiento de la estenosis aórtica. Tampoco se encontraron tasas superiores de ACV, en contraste con los resultados iniciales del PARTNER.

Los resultados de estos ensayos clínicos han producido cambios en las guías de práctica clínica y ello ha propiciado su inclusión tanto en las americanas como en las europeas. La conclusión derivada de estos estudios es la siguiente:

"La TAVI debe de ser considerada para el tratamiento de la estenosis aórtica severa sintomática, en pacientes no candidatos a cirugía de recambio aórtico y con esperanza de vida $>$ de 1 año, con la finalidad de mejorar su supervivencia y su capacidad funcional" se recomienda además una selección adecuada de pacientes con la finalidad rentabilizar al máximo los resultados ya que, la presencia de ciertas comorbilidades resta eficacia al procedimiento.

En pacientes con alto riesgo quirúrgico, la TAVI tiene resultados no inferiores al RVAo, por lo que se acepta como una alternativa válida a la cirugía.

\section{Registros sin distribución aleatoria}

En la tabla 2 se muestran los resultados de los principales registros. Agrupan a un total de 10.420 pacientes, bastante homogéneos y con resultados similares en términos de supervivencia y complicaciones más frecuentes.

El perfil de riesgo oscila entre un Euroescore logístico del 18 al 23\%, la mayor parte se realizan por vía transfemoral y la mortalidad a 30 días es, mayoritariamente por debajo del $5 \%$, aunque el en registro Alemán (697 pacientes) llega al 12,4\%. La mortalidad al año es similar, situándose entre el 15 y el $21,4 \%$, aunque en la mayoría esta próxima al $20 \%$. La incidencia de insuficiencia aórtica de grado moderado 0 severo se sitúa entre el 6,5 y el 24,5\%, posiblemente ligeramente más elevada con el dispositivo CoreValve. Este es un dato relevante porque un nivel de insuficiencia aórtica de grado moderado o severo, se asoció en el PARTNER a una mayor mortalidad.

El ACV se mantuvo en unos niveles siempre por debajo del $5 \%$, y generalmente alrededor del $3 \%$ que minimizan el impacto de esta grave complicación.

La incidencia de complicaciones vasculares ha disminuido muchísimo a medida que se han mejorado los dispositivos y disminuye, por tanto, la agresión vascular. En los últimos registros la incidencia de complicaciones vasculares se sitúa sistemáticamente por debajo del 7\%.

La implantación de Marcapasos permanentes debida al traumatismo de la válvula sobre el sistema de conducción es también un elemento importante que además influye de una forma relevante en los costos. Existe una clara diferencia entre ambas prótesis ya que en la Edwards está situada entre el 8-10\%. En la CoreValve, a pesar de cambios en el diseño y formas de implantación, se mantiene entre el 25 y 35\%. Al margen de casos anecdóticos, próximos a los 10 años de supervivencia, empieza a haber evidencia del comportamiento a largo plazo de las válvulas transcatéter, tanto por los resultados a 5 años de los estudios PARTNER, como los de registros de calidad. 
Tabla2. Principales registros observacionales

\begin{tabular}{|c|c|c|c|c|c|c|c|}
\hline Registro & Source XT & Alemán & Británico & Francés & Italiano & Iberoameric. & Advance \\
\hline $\mathrm{N}$ & 2.760 & 697 & 870 & 3.195 & 663 & 1.220 & 1.015 \\
\hline Sapien \% & 100 & 15,6 & 48 & 66,9 & & & \\
\hline CoreValve \% & & 84,4 & 52 & 33,1 & 100 & 100 & 100 \\
\hline Euroescore & 20,4 & 20,5 & 18,5 & 21,9 & 23 & 17,8 & 19,4 \\
\hline$\% \mathrm{TF}$ & 62,6 & 92,4 & 68,9 & 74,6 & 90 & 94,7 & 88,4 \\
\hline Muerte $30 \mathrm{~d}$. & 4,3 & 12,4 & 7,1 & 9,7 & 5,4 & 4,5 & 4,5 \\
\hline Muerte año & 19 & 20,2 & 21,4 & 24 & 15 & 17,9 & 17,9 \\
\hline $\mid A o>$ mod. & 6,2 & 17,5 & 13,6 & 16,5 & 21,2 & 24,5 & - \\
\hline $\mathrm{ACV} 30 \mathrm{~d}$ & 2,3 & 2,8 & 4,1 & 4,1 & 1,2 & 2,8 & 3,3 \\
\hline $\begin{array}{c}\text { Comp Vasc. } \\
30 \mathrm{~d}\end{array}$ & 7,3 & - & 6,3 & 4,7 & 2,1 & 3,9 & 10,9 \\
\hline MP & 8 & 39,3 & 16,3 & 15,6 & 16,6 & 24,8 & 26,3 \\
\hline
\end{tabular}

El seguimiento ecocardiográfico demuestra un comportamiento estable del funcionamiento de la válvula, con una reducción trivial del área valvular, un aumento no significativo del gradiente y sin regurgitación aórtica mayor que la reportada en el implante. La evaluación seriada con tomografía computarizada muestra ausencia de engrosamiento, calcificación o desestructuración valvular e integridad del stent de la prótesis. Estos datos se han corroborado en múltiples estudios, sin embargo, deben valorarse con cautela ya que se refieren a un número limitado de pacientes y además en muchos casos el seguimiento es necesariamente corto debido al fallecimiento por las comorbilidades asociadas.

\section{Evidencia en sustratos especiales}

\section{- Válvula aórtica bicúspide}

Inicialmente se consideró la válvula aórtica bicúspide como contraindicación para la TAVI, debido a que su anillo suele ser muy elíptico y también tener una distribución muy asimétrica del calcio, lo que favorece una Incidencia de insuficiencia aórtica mayor.

Sin embargo, la aorta bicúspide es la anomalía valvular más prevalente, afectando al 1-2\% de la población, de ahí la trascendencia del problema. Actualmente, a medida que ha aumentado la experiencia de los distintos grupos y han mejorado los prototipos valvulares, se considera solo como una contraindicación relativa.

La anatomía de estas válvulas se ha relacionado con una mayor incidencia de infraexpansión, mala posición y como consecuencia mayor grado de insuficiencia aórtica residual. También se ha sugerido la mayor tendencia a degeneración precoz de la prótesis, sin embargo se han publicado los resultados de muchos registros, en los cuales no hay diferencia significativa en mortalidad y aunque hay mayor incidencia de insuficiencia, esta no tiene repercusión en la supervivencia.

\section{-Insuficiencia aórtica}

Existe poca evidencia en el tratamiento de la insuficiencia aórtica. Solamente hay estudios aislados con el dispositivo
CoreValve en pacientes inoperables, en los que se han conseguido buenos resultados a expensas de una insuficiencia aórtica residual en el $21 \%$ y con necesidad de implantación de una segunda prótesis en el 19\% de casos (Roy et al), por tanto, en los pacientes con IAo inoperables se pueden obtener buenos resultados pero teniendo en cuenta estos dos factores.

\section{-Válvulas biológicas degeneradas/disfuncionantes}

Existe una gran cantidad de pacientes portadores de prótesis biológicas, la posibilidad de degeneración es un hecho real que se suele deber a calcificación, fractura, etc. Se estima que la incidencia de reintervención puede ser de hasta el 3\% a los 10 años.

Hasta ahora el estándar del tratamiento era la reintervención. Sin embargo la morbimortalidad de la misma oscila entre el $2-7 \%$ y en pacientes de alto riesgo puede superar el $20 \%$.

Por tanto, este es un nicho de pacientes que se pueden beneficiar claramente de la TAVI como alternativa terapéutica menos invasiva. El procedimiento (valve-in-valve) inicialmente contraindicado se viene realizando de forma prácticamente rutinaria y con excelentes resultados. Recientemente se han publicado los datos de un registro multinacional y multicéntrico con 459 pacientes con prótesis biológicas degeneradas tratados con TAVI. En este caso se pueden tratar pacientes con estenosis e insuficiencia. La supervivencia al mes es del 92,6\% y al año, del 83,2\%.

Los procedimientos valve-in-valve tienen una mayor posibilidad de mala posición y de obstrucción coronaria. La mala posición se debe al deslizamiento de la prótesis en el momento del despliegue o la dificultad para su ubicación en algunas prótesis, sin embrago los nuevos dispositivos hacen más predecible el despliegue de la válvula y su ubicación, por lo que su incidencia disminuye. La obstrucción de las arterias coronarias puede alcanzar el 3,5\%, muy superior a la descrita en las nativas, que está comprendida entre el 1 y el $1,5 \%$. La causa es el desplazamiento del material protésico 
al implantar la TAVI.

Teniendo en cuenta que es necesaria mayor experiencia en este campo, la TAVI se considera una alternativa a la reintervención quirúrgica para casos de alto riesgo en que incluso podría ser de elección.

\section{-Pacientes de riesgo intermedio}

Los resultados en este campo, sumamente atractivo, ya que supondrían un incremento importante en las indicaciones, son muy buenos pero se derivan solo de estudios observacionales. Actualmente se están realizando dos estudios con distribución aleatoria (SURTAVI y PARTNER II) que compararán la TAVI y el RVAo en pacientes con estenosis aórtica grave sintomática y riesgo quirúrgico intermedio (puntuación STS entre el 4 y el 8\%), sus resultados serán determinantes para aclarar este asunto y tendrán, seguramente implicaciones en las guías de práctica clínica.

Ya se comentaron los resultados del CoreValve US Pivotal Trial con la válvula autoexpandible Medtronic CoreValve ${ }$, que es un ensayo clínico randomizado que ha demostrado a medio plazo (2 años) la superioridad de la TAVI sobre el tratamiento quirúrgico convencional en términos de mortalidad con una incidencia de ACV similar.

Recientemente (ACC 15 de marzo 2015) se han comunicado Ios resultados a 30 días del PARTNER II (Kodali et al) comparando los resultados de la TAVI con la prótesis Edwards de última generación, Sapien III, en pacientes inoperables, de alto riesgo y de riesgo intermedio (Figura 5).

Aunque será necesario completar el seguimiento, los resultados en mortalidad e incidencia de accidente vascular cerebral son muy esperanzadores, ya que la muerte cardiovascular de la TAVI por vía transfemoral es menor del $1 \%$ y la incidencia de ACV del 1\%, muy inferiores a lo reportado hasta ahora.
Figura 5. Mortalidad y accidente vascular cerebral a 30 días con la válvula Sapien III de Edwards (S3HR=alto riesgo, S3i=riesgo intermedio)

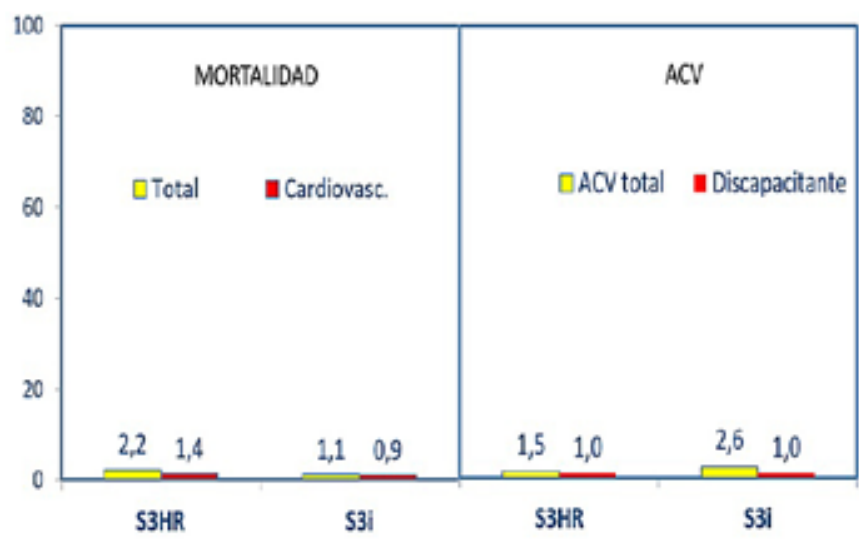

Otro aspecto muy importante (Figura 6) es la constatación de la mejoría importante de resultados con la evolución de la tecnología. El desarrollo de dispositivos de menor perfil, con menor regurgitación y mejor facilidad en el despliegue, junto con la mayor experiencia de los cardiólogos intervencionistas ha hecho que la mortalidad se la implantación se haya reducido prácticamente 6 veces, del 6,3 al 1\%, desde la primera a la última generación de la prótesis Edwards. Estos resultados son similares con la CoreValve.

La Sapien III se acompaña además, de una disminución muy importante de las complicaciones más frecuentes (Tabla 3), fundamentalmente en lo referido a complicaciones vasculares y sangrado, la incidencia de rotura aórtica, la baja incidencia de oclusión coronaria, menor del 0,5\% y una baja necesidad de implantación de Marcapasos permanentes post procedimiento, que con esta prótesis es del 10\%, aunque con las autoexpandibles está más próxima al 30\%.

Figura 6. Evolución de la mortalidad con las distintas generaciones de la prótesis de Edwards

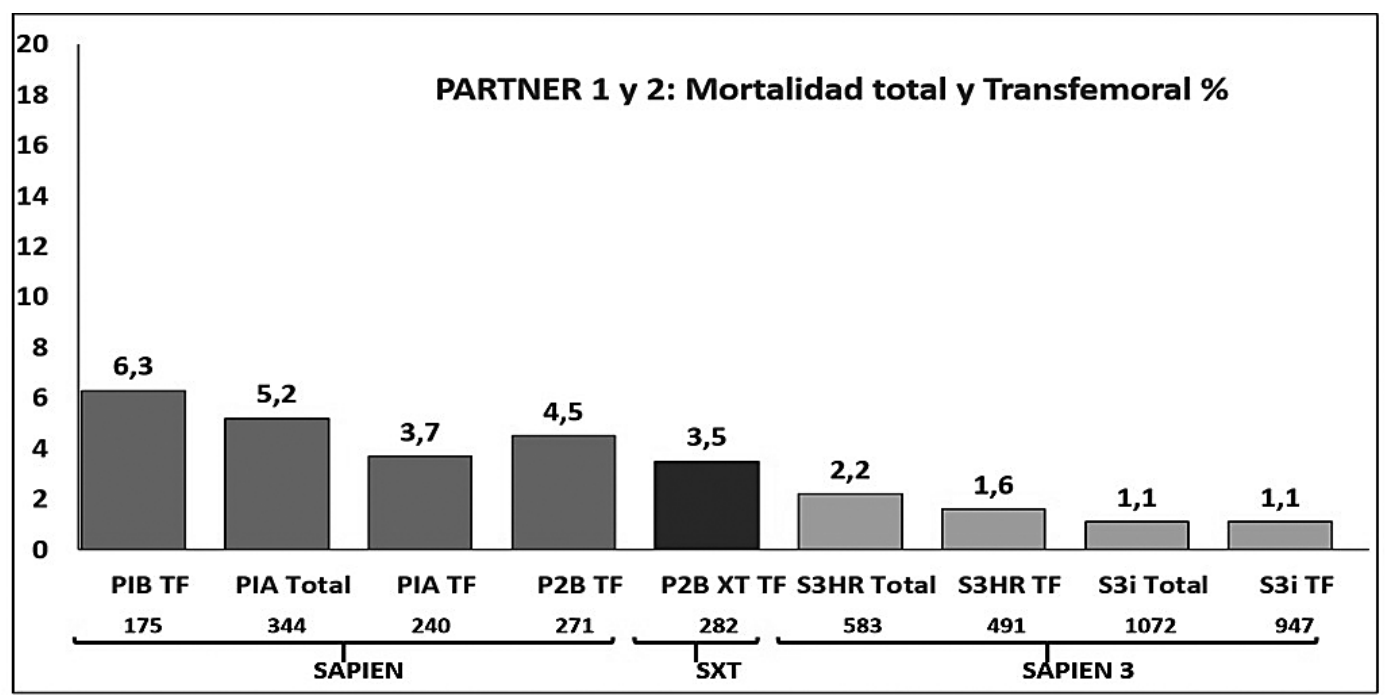

(PIB=PARTNER I; P2=PARTNER II; S3HR=Sapien III alto riesgo; S3i=Sapien II riesgo intermedio; SXT=Sapien XT) 
Tabla3. Incidencia de las complicaciones más frecuentes con la Sapien III, totales y por acceso transfemoral (TF)

\begin{tabular}{|l|c|c|c|c|}
\hline Eventos (\%) & $\begin{array}{c}\text { S3HR } \\
\text { Total } \\
(n=583)\end{array}$ & $\begin{array}{c}\text { S3HR } \\
\text { TF } \\
(n=491)\end{array}$ & $\begin{array}{c}\text { S3i } \\
\text { Total } \\
(n=1076)\end{array}$ & $\begin{array}{c}\text { S3i } \\
\text { TF } \\
(n=951)\end{array}$ \\
\hline Complic. Vasculares mayores & 5.0 & 5.3 & 5.6 & 5.9 \\
\hline Sangrado con riesgo vital & 6.3 & 5.5 & 5.4 & 4.4 \\
\hline Rotura del anillo & 0.3 & 0.2 & 0.2 & 0.2 \\
\hline Infarto Agudo de Miocardio & 0.5 & 0.4 & 0.3 & 0.3 \\
\hline Obstruccion coronaria & 0.2 & 0 & 0.4 & 0.4 \\
\hline Fracaso renal agudo & 1.0 & 0.8 & 0.5 & 0.3 \\
\hline Necesidad de MP permanente & 13.0 & 13.2 & 10.1 & 10.4 \\
\hline Endocarditis & 0.2 & 0.2 & 0.1 & 0.1 \\
\hline
\end{tabular}

\section{Complicaciones/Limitaciones de la TAVI}

El conocimiento de las posibles complicaciones y limitaciones de la TAVI es muy importante para el cardiólogo intervencionista y el clínico. A pesar de los avances en el diseño, no se ha conseguido hacerlas desaparecer, aunque han disminuido de forma sustancial. El conocimiento de las mismas, su tratamiento y sobre todo su prevención son capitales para una indicación adecuada, sobre todo teniendo en cuenta que la prevalencia de comorbilidades en los pacientes candidatos en muy elevada.

Estas complicaciones, potencialmente muy graves, no son infrecuentes por lo que es necesario conocer los factores predisponentes y las estrategias de prevención y tratamiento.

Los avances en el desarrollo de los dispositivos y la mayor experiencia de los operadores han contribuido a reducir significativamente la prevalencia de algunas, y seguramente en el futuro disminuyan todavía más.

A continuación se comentaran las principales complicaciones inherentes a la TAVI y que de alguna forma representan las limitaciones de la técnica.

\section{Complicaciones vasculares}

Aunque ha disminuido de forma importante el perfil de los dispositivos, dada la edad avanzada de los pacientes, no es infrecuente que los dispositivos tengan que avanzar por un árbol arterial ateromatoso, calcificado y además la necesidad de hemostasia del punto de acceso hace que la incidencia de complicaciones y sangrado no sea despreciable. Además, estas complicaciones son importantes por el impacto en el pronóstico.

El abordaje transfemoral es el más frecuente y casi exclusivamente percutáneo ya que la técnica con disección y reparación quirúrgicas se utilizan de forma poco habitual. Las complicaciones más frecuentes son las relacionadas con el lugar de acceso pero pueden ocurrir a otros niveles del árbol vascular.
Los problemas en el lugar de acceso son muy variables: disección, obstrucción, estenosis, perforación o rotura, fístulas AV, seudoaneurisma, hematoma y síndrome compartimental, daño de un nervio o fallo del dispositivo hemostático que pueden producir la muerte del paciente, sangrado mayor o menor, isquemia visceral, daño neurológico permanente 0 la necesidad de intervención quirúrgica 0 endovascular no previstas.

En el PARTNER (2007 - 2009) presentaron complicaciones mayores el $15 \%$ de los pacientes y complicaciones menores, el $12 \%$. En otros registros más recientes la tasa de complicaciones mayores fue entre el 3 y el $5 \%$. Las complicaciones vasculares mayores se asocian con más sangrado, necesidad de transfusión y con un aumento de hasta 4 veces en la mortalidad a 30 días. Las complicaciones vasculares menores no influyen el pronóstico.

Se han sugerido como factores predictivos de complicaciones vasculares la enfermedad vascular periférica, la calcificación, la tortuosidad vascular, diámetros femorales pequeños, la experiencia del operador y el sexo femenino. Una selección adecuada, valoración anatómica, y una técnica cuidadosa de punción y hemostasia ayudad a prevenir las complicaciones vasculares.

\section{Insuficiencia aórtica residual}

La insuficiencia aórtica residual, generalmente paravalvular, es muy frecuente en la TAVI. La insuficiencia aórtica residual de algún grado ocurre en más del $50 \%$ de los pacientes, si se considera solo la de grado $\geq 2$, la frecuencia disminuye al 10 y el 20\%, según la prótesis.

En el estudio PARTNER se objetivó regurgitación paravalvular moderada 0 grave en el $12 \%$. Además, la regurgitación residual se asoció a una menor supervivencia, de ahí la importancia de esta complicación que se suele deber a la aposición incompleta debida a mala selección del tamaño 0 expansión inadecuada, por calcificación importante 0 liberación en una posición incorrecta.

La prevención requiere una valoración anatómica exhaustiva previa al implante, mediante una buena modalidad de imagen (Ecografía tridimensional o tomografía axial computarizada) para la medición exacta del anillo aórtico. Es necesaria una técnica cuidadosa para la adecuada altura del implante y expansión de la prótesis.

Los dispositivos han tenido sucesivas mejoras de diseño de la propia válvula y del sistema de liberación que han conseguido una mayor predictibilidad de la zona de implantación y mejor aposición. La prótesis SAPIEN III, y debido a un faldón para prevenir la insuficiencia paravalvular, ha disminuido la incidencia de insuficiencia moderada o severa al 3,9\% (0,1\% severa). Probablemente futuros diseños podrían ayudar a controlar aún más este problema mejorando la aposición, el reposicionamiento o la eventual recaptura del dispositivo.

\section{Trastornos de la conducción}

La TAVI se asocia con frecuencia a trastornos de la conducción como el BCRl y el bloqueo AV, ocasionalmente con 
necesidad de implantación de un MP permanente. Aproximadamente el $45 \%$ sufren BRI y la necesidad de implantar un marcapasos varía entre el 4 y el 33\%, según el tipo de prótesis.

La estenosis aórtica se asocia con trastornos de la conducción, de hecho la cirugía de recambio valvular tiene una tasa de implantación de marcapasos comprendida entre el 3 y el 8\%. La posibilidad de desarrollo de los trastornos de la conducción AV tras la TAVI se ve favorecida por la íntima relación anatómica entre la válvula aórtica y el sistema de conducción, nódulo Auriculo Ventricular, salida del haz de Hiss y rama izquierda que está muy próxima a las valvas no coronariana y coronariana derecha. El mecanismo más probable es la compresión mecánica por la estructura de la prótesis que puede producir inflamación o traumatismo del mismo.

La implantación de la prótesis autoexpandibles se asocia con una mayor tasa de implantaciones, superior al 10\% y hasta del $49 \%$, mientras que con la de prótesis de Edwards es inferior al 10\%. Las razones de esta diferencia pueden deberse a varias causas, por un lado el diferente mecanismo de liberación, autoexpandible en la CoreValve y expandible con balón en la Sapien. Por otro, las diferencias estructurales de la prótesis como la composición, nitinol y acero respectivamente, y la diferente longitud ya que la CoreValve es más larga y penetra más en el tracto de salida, lo que facilita la lesión del sistema de conducción.

Los factores predictivos que se asocian más claramente con el desarrollo de bloqueo AV son la implantación baja, sobretodo de la CoreValve, y el bloqueo de rama derecha preexistente. EL BRI no se asocia con mayor necesidad de MP.

\section{Accidente cerebrovascular}

Aunque el accidente vascular cerebral (ACV) es infrecuente es posiblemente la complicación más devastadora y temida de la TAVI debido al importante impacto en la calidad de vida y la supervivencia.

La manipulación de los de los catéteres en el arco aórtico y la válvula aórtica pude producir la embolización de material ateromatoso o trombótico. Con algunas técnicas de imagen se ha comprobado que durante la TAVI se produce embolización cerebral asintomática muy frecuentemente, entre el 65 y 85\%, sin embargo la repercusión clínica es mucho menor. La experiencia inicial (estudio PARTNER) mostro una incidencia de ACV del 6,7\%. En estudios posteriores, las tasas registradas han sido menores, y un metanálisis de 2012 cifra la frecuencia de ACV en el 3,3\%. Registros más recientes, presenta tasas, aún menores, del 1,8\%.

La presencia de ateromatosis aórtica, la posdilatación de la prótesis y la manipulación excesiva se han relacionado con un riesgo aumentado de ACV, por tanto la prevención se basa en minimizar la agresión vascular y valvular durante el procedimiento. El mejor diseño de las válvulas y la mayor experiencia de los operadores también contribuyen a la disminuir la incidencia de esta complicación. Como se comentó previamente con los nuevos dispositivos, Sapien III en concreto, los resultados iniciales muestran una incidencia realmente baja de ACV incapacitante, de solo un 1\% Se han ensayado diversos dispositivos de protección embólica, pero su eficacia no está claramente establecida. Insuficiencia renal

La incidencia de insuficiencia renal post-TAVI es variable según distintas series. Se estima que la incidencia de daño renal puede estar entre el 10 y el 20\%, sin embargo, la tasa de daño renal importante en un 4-8\% y entre el 1,5 y $5 \%$ de los pacientes necesitan diálisis.

Es una importante complicación debido al aumento de mortalidad. La etiología es multifactorial, y se relaciona con la edad, HTA, diabetes, enfermedad vascular, ateroembolias y toxicidad del contraste. La insuficiencia renal aguda postTAVI duplica el riesgo de muerte y puede oscilar entre el 20 y el $50 \%$.

La prevención del daño renal post-TAVI se basa en las medidas habituales utilizadas en procedimientos intervencionistas.

\section{Complicaciones poco frecuentes}

Rotura del anillo o de la raíz aórtica

Es una complicación muy rara, menos del 1\%. La calcificación extensa y circunferencial del tracto de salida, del anillo y raíz aórtica, el sobredimensionado de los balones 0 prótesis son las causas más frecuentes. La principal manifestación clínica es un deterioro hemodinámico inmediato debido al taponamiento cardiaco.

Esta complicación es menos frecuente en las prótesis autoexpandibles, y ha disminuido desde que se realizan las mediciones más exactas con TAC y ECO 3D.

Oclusión coronaria

Es una complicación infrecuente $(<1 \%)$, pero con consecuencias potencialmente muy graves. La causa pude ser el desplazamiento de las valvas nativas o protésicas sobre el ostium coronario. En el $80 \%$ de los casos ocurre en la coronaria izquierda y es más frecuente en pacientes con salida baja de las arterias coronarias, y aorta estrecha. Su incidencia también es mayor en los procedimientos "Valve in valve".

El tratamiento es la desobstrucción inmediata mediante angioplastia coronaria, con una tasa de éxito mayor del $80 \%$. La valoración anatómica previa, permite conocer los casos con salida muy baja del ostium coronario y una aorta estrecha, y es la mejor estrategia de prevención. Se ha propuesto la protección con una guía intracoronaria en casos de alto riesgo.

\section{Perforación cardiaca}

Puede ocurrir en el ventrículo derecho, debido al cable de marcapasos y en el ventrículo izquierdo por el uso de dispositivos muy agresivos. El taponamiento cardiaco se produce en el $2 \%$. El drenaje pericárdico suele solucionar el problema, aunque puede ser necesaria la reparación quirúrgica. La sospecha ante un cuadro de hipotensión inexplicada es muy importante. 


\section{Selección de candidatos/Indicaciones}

Dadas las dificultades para el análisis en los síntomas en estos pacientes, la selección de pacientes candidatos debe de basarse en varios puntos importantes:

1. Valoración clínica que confirme los síntomas y que estos se correlacionan con la estenosis aórtica.

2. Análisis de otras comorbilidades que influyan en el pronóstico y que puedan afectar la calidad y la cantidad de vida. Capacidad del paciente para su propia autonomía ya que cuando está muy disminuida el beneficio en mínimo.

3. Estudio de la fragilidad ya que las posibles complicaciones tienen muchísima más gravedad en los pacientes frágiles.

4. Estudio anatómico de la válvula la aorta, y las arterias iliacas para considerar una TAVI y decidir la vía de acceso.

Las guías, europea y americana, de práctica clínica dan un papel central al equipo cardiológico (Heart Team) en la valoración individualizada de la mejor opción de tratamiento para los pacientes con estenosis aórtica inoperable 0 de alto riesgo quirúrgico. Este equipo debe evaluar los riesgos individuales, factibilidad técnica y el abordaje más apropiado. También se analizarán las contraindicaciones clínicas y anatómicas. La expectativa de vida debe de ser $\geq 1$ año y tiene que existir probabilidad de mejoría de calidad de vida.

Se propone como indicación para TAVI el alto riesgo quirúrgico con EuroSCORE logístico > 20\% o un puntuación superior al $10 \%$ por el sistema de la Society of Thoracic Surgeons (STS). En ciertas situaciones clínicas, como aorta en porcelana, injertos aortocoronarios previos, radiación torácica, sin alto riesgo quirúrgico, se puede plantear TAVI como una alternativa a la cirugía. En estos casos la decisión debe tomarse en el Heart Team. Los pacientes con riesgo quirúrgico intermedio quedan excluidos de este tratamiento a la espera de los resultados de los estudios en marcha,

\section{Situación actual}

La publicación de los resultados ha provocado un interés cada vez más importante en esta tecnología, como lo demuestra el número de publicaciones científicas que ha crecido de forma exponencial en los últimos años (Figura 7).

Los datos de implantación que se presentan a continuación están obtenidos de una base de datos que registra la información de 15 países de la UE. Se muestran la evolución de implantes (Figura 8), a la izquierda el total de implantes desde el 2009 al 1013 y a la derecha el total por año en el mismo periodo. Como se puede observar se ha multiplicado por 4 el número de implantes anuales y por 18 el número total de implantes.
Figura 7. Publicaciones/año TAVI

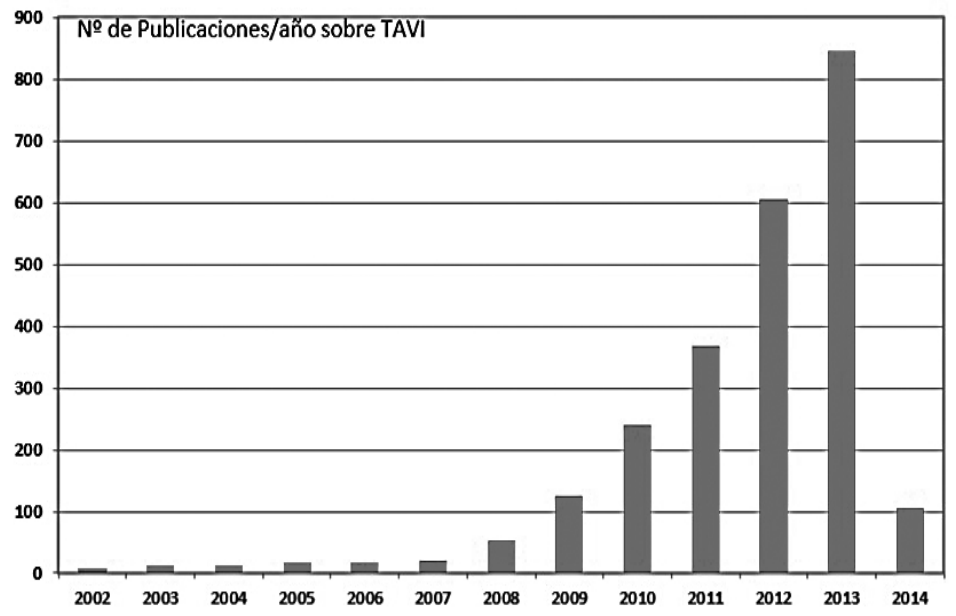

En la figura 9 se ve la tasa de implantaciones por millón de habitantes, en el global de los 15 países de la EU que se analizan. Según esto, actualmente la tasa de implantes se sitúa en casi 54 por millón, con un aumento progresivo, pero con tendencia a la estabilización en los últimos años.

Sin embargo existen unas grandes variaciones entre países (Figura 10), dependientes de varios factores entre los cuales, posiblemente los recursos financieros y la organización de los distintos sistemas de salud tengan importancia.

Estas diferencias están comprendidas entra los 118/millón de Alemania y las 9 de Portugal, España se sitúa en la tercera posición global por la cola con 18,4 por millón aunque con grandes variaciones entre hospitales y comunidades. La media de la comunidad europea es de 53.6, pero si se excluye a Alemania la tasa cae a 37,7.

Según los datos comentados previamente, la posición global en España está entre las más bajas de Europa, sin embargo según los datos del registro nacional de TAVI, promovido por la Sección de Hemodinámica de la SEC, también existe una gran dispersión en la utilización de esta terapéutica en las distintas comunidades autónomas en general, y entre unidades de hemodinámica en particular. Lamentablemente, a pesar de que la sección de Hemodinámica está realizando un registro, al ser voluntario, solo una pequeña parte de los casos realizados en España son incluidos en el mismo y la

Figura 8. Experiencia CHUAC. Cambios en la clase funcional

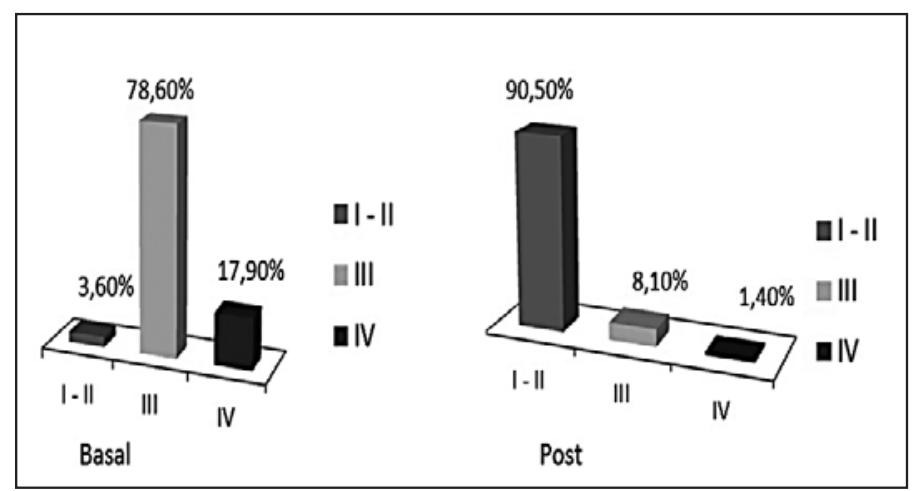


Figura 9. Evolución de implantes en la Unión Europea total y por año
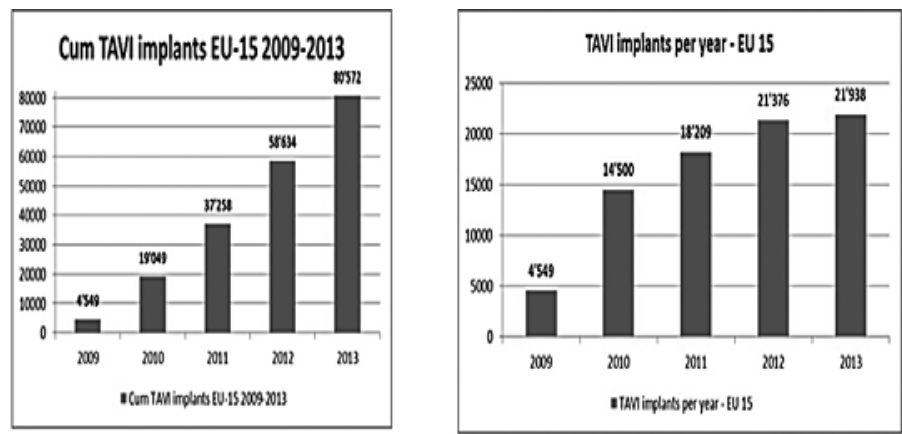

Figura 10. Tasa de implantación/millón de habitantes en la UE

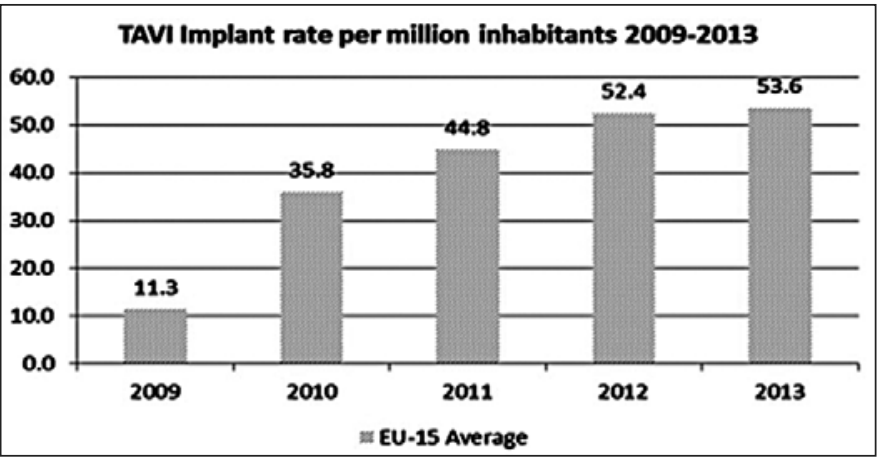

información por esta vía no solo es inexacta, muchos centros no remiten su casuística, sino que introduce numerosos elementos de confusión.

Son mucho más fiables los datos suministrados por las compañías, ya que llevan un registro exhaustivo de los implantes que se realizan en todos los hospitales del estado. Según estos datos existen muchas diferencias en la tasa de implantación por comunidad (Figura 11).

En el año 2013 se implantaron en total 1038 prótesis en España, lo que representa aproximadamente 28/millón habitantes. De estas 433 fueron autoexpandibles (CORE VALVE, Medtronic) y 605 expandibles con Balón (Edwards), de estas 443 por vía transfemoral, 137 transapicales y 25 transaórticas. Los rangos de implantación van de las 54/ millón de Cantabria y País Vasco a las 5/millón de Baleares. Galicia se sitúa en una media de 47/ millón con 42 implantes en Coruña, 40 implantes en Santiago y 23 en el área sur lo que representa una tasa de implantación de 42 en Coruña y área norte, casi 80 en el área centro y 20 en el sur. Es decir, dentro de nuestra comunidad existe una clara dispersión en la tasa de implantación, seguramente debida a una discrepancia en los criterios de selección.
Es probable que la penetración en nuestro país se incremente y se sitúe en cifras más próximas a los 60-70 implantes por millón, manteniendo las indicaciones actuales y con tendencia clara a que en los próximos 5 años se amplíen las mismas, una vez que se confirme la durabilidad de la prótesis y la favorable evolución clínica en cohortes más amplias de pacientes.

\section{Experiencia CHUAC}

Nuestra unidad inició esta técnica en el último trimestre de 2008, fuimos uno de los primeros de España y la primera prótesis de Galicia se implantó en nuestro laboratorio. Actualmente tenemos una experiencia cumulada superior los 200 implantes con una cifra próxima a 50 implantes anuales.

Las características basales de nuestra serie no difieren de las aceptadas internacionalmente. Se realiza una consulta específica para estos pacientes donde se evalúa a los potenciales candidatos remitidos desde otras unidades clínicas 0 áreas

También se realiza el seguimiento de los pacientes tratados por lo que sirve de control de calidad.

Figura 11. Tasa de implantación por países de la UE. A la derecha sin Alemania
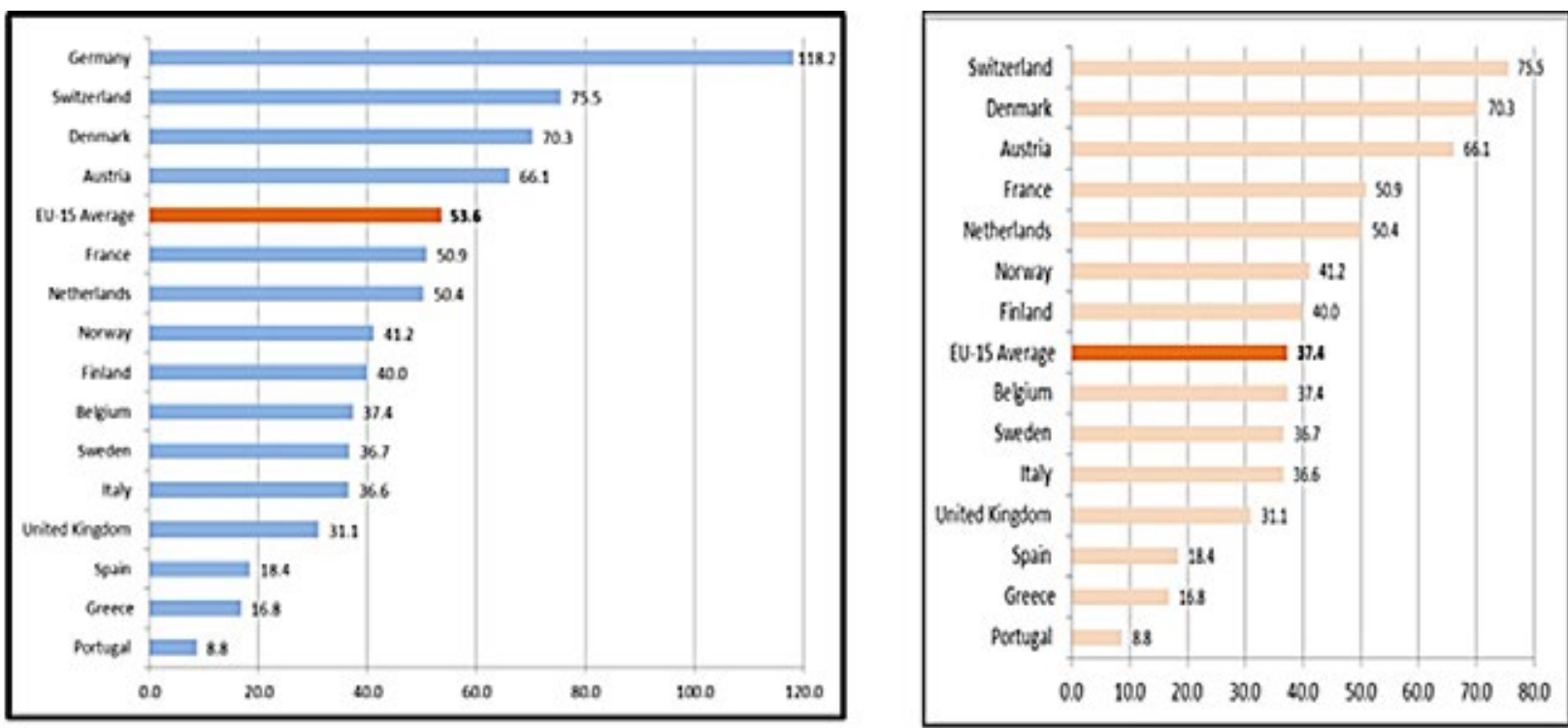


\section{Resultados}

Los resultados globales pueden verse en la tabla 4 . Hay una incidencia de complicaciones vasculares mayores del 7,6 \%, que se ha reducido drásticamente desde la disminución de perfil del dispositivo. Otro hecho importante es que, actualmente todas estas complicaciones se resuelven en la sala sin concurso de otros especialistas. Con el advenimiento de la nueva generación (Sapien III) han disminuido todavía más, ya que el calibre es menor (14F).

Tabla 4. Experiencia CHUAC. Complicaciones y resulyados

\begin{tabular}{|c|c|c|c|}
\hline Complicaciones: & & $\begin{array}{l}\text { Estancia } \\
\text { (Días) }\end{array}$ & $5,0 \pm 3,66$ \\
\hline Complicaciones vasculares & & Grado de IAo & \\
\hline Mayores & $12(7,6 \%)$ & $0-1$ & $116(73,4 \%)$ \\
\hline Totales & $30(19,0 \%)$ & 2 & $25(15,8 \%)$ \\
\hline Necesidad de marcapasos & $5(3,2 \%)$ & 3 & $3(1,9 \%)$ \\
\hline Necesidad de diálisis & $4(2,5 \%)$ & Mortalidad & \\
\hline$A C V$ & $2(1,3 \%)$ & Muerte intra-procedimiento & $7(4,4 \%)$ \\
\hline Malposición de la prótesis & $3(1,9 \%)$ & Mortalidad a 30 dias & $9(5,7 \%)$ \\
\hline Oclusión coronaria & $6(3,8 \%)$ & Mortalidad a 1 año & $19(12,0 \%)$ \\
\hline
\end{tabular}

En cuanto a otras complicaciones, es significativa la baja tasa de necesidad de implantación de marcapasos, de ictus y la tasa de oclusión coronaria, superior a los registros y posiblemente relacionada con características propias de nuestros pacientes. En todos los casos, esta complicación; se solucionó en la sala y no se acompañó de efectos adversos. La incidencia de insuficiencia aórtica moderada severa fue menor del 2\%, la mortalidad a 30 días del $5,75 \%$ y al año del $12 \%$ que son inferiores a las reportadas, alrededor del $10 \%$ y del $25 \%$ respectivamente.
Además, los buenos resultados en mortalidad se acompañaron de una mejoría clínica muy relevante con cambios importantes de la clase funcional que implican, en la mayoría de pacientes, un aumento muy importante en su calidad de vida (Figura 12). En concreto, los pacientes en clase funcional III o IV pasan del 96,5 al 9,5\% lo que representa un cambio radical en su situación.

Todo esto sin un impacto relevante sobre la actividad quirúrgica global que no solo se mantiene, sino que parece que se ha incrementado debido a que, no solo se tratan mediante prótesis transcatéter o cirugía convencional a los pacientes añosos con contraindicación o alto riesgo quirúrgico, sino también a un grupo de pacientes que antes no eran operados y ahora lo son. Este es un fenómeno que se repite en todos los equipos quirúrgicos de España y Europa pone de manifiesto que el $40 \%$ de pacientes que no recibían tratamiento quirúrgico convencional era poco justificable.

\section{Selección de pacientes}

La posición del Servicio de cirugía cardiaca esta consensuada con el servicio de cardiología y el acuerdo de los dos servicios es total. Consideramos buenos candidatos para TAVI a los pacientes que cumplen las siguientes características:

- Presencia de estenosis valvular aórtica sintomática por angina, disnea o síncopes.

- Pacientes de alto riesgo quirúrgico, o considerados 'inoperables' por su edad o comorbilidades.

- Limitación funcional fundamentalmente por su patología cardíaca, en los que se considera a priori, que la implantación de una prótesis valvular va a mejorar significativamente su calidad de vida y su capacidad funcional.

Que son, en esencia, los recogidos en las guías de práctica clínica americanas y europeas, cuya última versión corresponde al año 2014 (Tabla 5).

Figura 12. Tasa de implantación España 2013

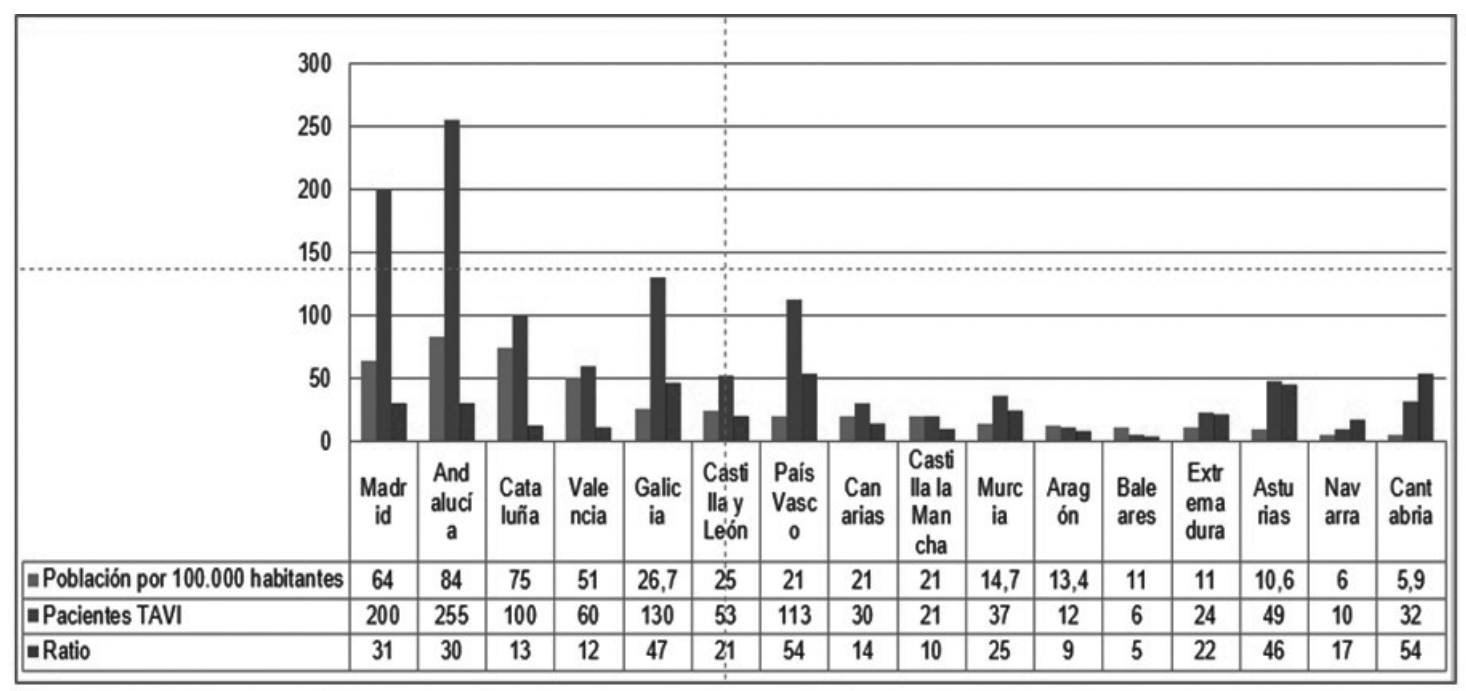


Tabla 5. Guías AHA 2014. Indicaciones para el tratamiento de la estenosis aórtica severa

\begin{tabular}{|c|c|c|}
\hline Recomendaciones AHA en la elección de Cirugía o TAVI & Clase & Nivel \\
\hline Serecomienda sustitucón aórtica quirúrgica en pacientes de riesgo bajo o intermedio. & | & A \\
\hline $\begin{array}{l}\text { En pacientes de alto riesgo quirúrgico candidatos para TAVI l Heart Team consensuara la } \\
\text { mejor opción terapeútica. }\end{array}$ & | & C \\
\hline $\begin{array}{l}\text { Serecomienda TAVl en pacientes de riesgo quirúrgico prohibitivo con una supervivencia } \\
\text { estimada > de } 12 \text { meses. }\end{array}$ & । & B \\
\hline La TAV es una alternativa razonable a la cirugia de alto riesgo quirúrgico & $\|_{a}$ & $B$ \\
\hline $\begin{array}{l}\text { La valwuloplastia aórtica se puede considerar como puente a la cirugia o TAVI I n pacientes } \\
\text { con estenosis aórtica severamente sintomáticos. }\end{array}$ & $\| b$ & C \\
\hline $\begin{array}{l}\text { La TAVI no se recomienda cuando las comorbilidades existentes pueden superar los } \\
\text { beneficios esperados }\end{array}$ & $\|$ & $B$ \\
\hline
\end{tabular}

La razón final de este tratamiento es una mejora de calidad/ cantidad de vida en pacientes gravemente limitados por su valvulopatía, y por ello nuestro grupo sólo lo considera en pacientes con limitación severa por síncopes, disnea 0 angina (NYHA III o IV). Los pacientes poco sintomáticos o aquellos muy limitados por patología extracardíaca (patología pulmonar, osteoarticular, déficits neurológicos severos....), o los pacientes con aceptable riesgo quirúrgico no se consideran inicialmente buenos candidatos para TAVI.

Los índices de riesgo quirúrgico se calculan en todos los pacientes. Aunque estos índices, basados en ecuaciones de regresión, describen adecuadamente el riesgo en poblaciones de pacientes con determinadas características clínicas, son menos útiles de cara a valorar pacientes individuales. Por ello, aunque los consideramos de ayuda, no utilizamos estas escalas de forma absoluta para indicar uno u otro tratamiento.

El origen de los pacientes es muy heterogéneo, y proceden fundamentalmente de los servicios de cardiología y medicina interna del CHUAC, los hospitales de referencia y del servicio de cirugía cardíaca del CHUAC, con un esquema flexible en el flujo de pacientes, que permite la valoración tanto de pacientes ambulatorios como hospitalizados, así como la evaluación conjunta de los pacientes en sesiones médico-quirúrgicas y la posibilidad de reevaluación de los pacientes si cambian las circunstancias clínicas.

El programa es muy restrictivo y solo se ofrece a los pacientes que claramente se pueden beneficiar, un 37\% del total de los evaluados. Es relevante que en la espera se produce un $8 \%$ de éxitus (lo que ilustra la gravedad de los mismos), y que una pequeña proporción se redirigen a recambio valvular convencional.

La eficacia de un programa de TAVI y sus resultados clínicos se basan en varios elementos fundamentales:

1. Consenso en las indicaciones entre los diversos profesionales implicados: cirujanos, cardiólogos clínicos, aneste- sistas, cardiólogos ecocardiografistas e intervencionistas (Heart Team) que asegura la mejor técnica y el ajuste de las indicaciones.

2. Selección exhaustiva de candidatos y con carácter restrictivo: Realizada en una consulta específica de valvulopatías, con el consenso del servicio de Cirugía Cardiaca.

3. Seguimiento sistemático de pacientes: Realizado en la misma consulta y que sirve para el análisis de resultados a corto, medio y largo plazo así como de control de calidad para validar que el esfuerzo económico se correlaciona con los resultados clínicos.

El procedimiento de TAVI es muy exigente y requiere un entrenamiento importante que implique a intervencionistas expertos y un equipo multidisciplinar que incluye a cirujanos cardiacos, anestesistas y cardiólogos expertos en imagen cardiaca y, de acuerdo con las guías de práctica clínica debería de realizarse en centros con amplia experiencia, volumen y con acceso inmediato a cirugía cardiaca en caso de complicaciones.

\section{Futuro}

Actualmente se acepta por las guías de práctica clínica que, la TAVI es el tratamiento de elección para los pacientes inoperables y una clara alternativa para los de alto riesgo quirúrgico. Otras indicaciones tiene que ser discutidas y consensuadas por el Heart Team.

La técnica ha crecido exponencialmente en pocos años debido sus buenos resultados, sólidamente documentados. Esto hace previsible la ampliación de indicaciones a pacientes con riesgo quirúrgico intermedio o bajo y ciertos casos de insuficiencia aórtica. Su uso en patologías, inicialmente consideradas contraindicación, como la aorta bicúspide y prótesis degeneradas, ya se realiza de forma prácticamente rutinaria. Esta ampliación se producirá en base a varios factores:

1. Los prometedores resultados de los estudios en marcha en pacientes de riesgo intermedio

2. Mejora de los criterios de selección de los pacientes.

3. Mejora de aspectos procedimentales, como consecuencia del mayor conocimiento y experiencia de los grupos.

4. Mejora de los dispositivos con reducción del calibre, mejora de los sistemas de liberación y diseño con la finalidad de aumentar la seguridad y eficacia y disminuir las complicaciones inherentes a la técnica (reducción del trauma vascular, IAo residual y MP).

Esta mejora de los dispositivos hará que la vía de acceso femoral sea de elección en la mayor parte de los casos, mientras que otras vías quedarán restringidas a los pocos en que esta no sea factible. El desarrollo de nuevas válvulas probablemente permitirá la elección de la más adecuada para cada caso y aportaran la posibilidad de recapturar, reposicionar 0 retirarlas cuando el resultado no se considere óptimo. 
Un aspecto crítico y con un impacto muy importante para la expansión de la técnica, es el elevado precio de los dispositivos (del orden de $20.000 €$ ). La estimación de necesidades hace practicante inviable la financiación por un sistema como el español. No es previsible que corto plazo se produzca esta disminución del costo, agravado por un sistema que no considera el costo por proceso, sino solamente el presupuesto y gasto anual.

En conclusión, la TAVI ha revolucionado el tratamiento de la estenosis aórtica. Permite tratar a pacientes que antes no recibían tratamiento y se considera una alternativa a la cirugía para los de alto riesgo. Los excelentes resultados obtenidos, presagian que se utilizara rápidamente en pacientes de menor riesgo y nuevas indicaciones.

La disminución de complicaciones harán el procedimiento más seguro, predictivo y poco traumático, por lo que en los próximos años, la evolución de la técnica sea probablemente la misma que en su momento tuvo la angioplastia coronaria. Los buenos resultados, mínima agresión y menor estancia hospitalaria generaran una demanda cada vez mayor por parte de los pacientes y médicos referentes.

\section{Bibliografía}

1. Cribier A, Eltchaninoff H, Bash A, Borenstein N, Tron C, Bauer F, et al. Percutaneous transcatheter implantation of an aortic valve prosthesis for calcific aortic stenosis: first human case description. Circulation. 2002;106:3006-8.

2. Leon MB, Smith CR, Mack M, Miller DC, Moses JW, Svensson LG, et al; PARTNER Trial Investigators. Transcatheter aortic-valve implantation for aortic stenosis in patients who cannot undergo surgery. N Engl J Med. 2010;363:1597-607.

3. Smith CR, Leon MB, Mack MJ, Miller DC, Moses JW, Svensson LG, et al; PARTNERTrial Investigators. Transcatheter versus surgical aortic-valve replacement in highriskpatients. N Engl J Med. 2011;364:2187-98.

4. Svensson LG, Blackstone EH, Rajeswaran J, Brozzi N, Leon MB, Smith CR, et al. Comprehensive analysis of mortality among patients undergoing TAVR: results of the PARTNER trial. J Am Coll Cardiol. 2014;64:158-68.

5. Hahn RT, Pibarot P, Stewart WJ, Weissman NJ, Gopalakrishnan D, Keane MG, et al. Comparison of transcatheter and surgical aortic valve replacement in severe aortic stenosis: a longitudinal study of echocardiography parameters in cohort A of the PARTNER trial (Placement of Aortic Transcatheter Valves). J Am Coll Cardiol. 2013;61:2514-21.

6. Adams DH, Popma JJ, Reardon MJ. Transcatheter aortic-valve replacement with a self-expanding prosthesis. N Engl J Med. 2014;70:1790-8.

7. Linke A, Wenaweser P, Gerckens U, Tamburino C, Bosmans J, Bleiziffer S, et al; ADVANCE study Investigators. Treatment of aortic stenosis with a self-expanding transcatheter valve: the International Multi-centre ADVANCE Study. Eur Heart J. 2014;35:2672-84.

8. Adams DH, Popma JJ, Reardon MJ. Transcatheter aortic-valve replacement with a self-expanding prosthesis. N Engl J Med. 2014;370:1790-8.

9. Leon MB, Piazza N, Nikolsky E, Blackstone EH, Cutlip DE, Kappetein AP, et al. Standardized endpoint definitions for transcatheter aortic valve implantation clinical trials. A consensus report from the Valve Academic Research Consortium. J Am Coll Cardiol. 2011;57:253-69.

10. Kappetein AP, Head SJ, Généreux P, Piazza N, Van Mieghem NM, Blackstone EH, et al. Updated standardized endpoint definitions for transcatheter aortic valve implantation: the Valve Academic Research Consortium-2 consensus document. J Thorac Cardiovasc Surg. 2013;145:6-23.

11. Webb J, Gerosa G, Thierry Lefèvre JT, Leipsic J, Spence M, Thomas M, et al. Multicenter evaluation of a next-generation balloon-expandable transcatheter aortic valve. J Am Coll Cardiol. 2014;64:2235-43.
12. Vahanian A, Alfieri O, Andreotti F, Antunes MJ, Barón-Esquivias G, Baumgartner H, et al. Guidelines on the management of valvular heart disease (version 2012). Eur Heart J. 2012;33:2451-96.

13. Bax JJ, Delgado V, Bapat V, Baumgartner H, Collet JP, Erbel R, et al. Open issues in transcatheter aortic valve implantation. Part 1: patient selection and treatment strategy for transcatheter aortic valve implantation. Eur Heart J. 2014;35:2639-54.

14. Bax JJ, Delgado V, Bapat V, Baumgartner H, Collet JP, Erbel R, et al. Open issues in transcatheter aortic valve implantation. Part 2: procedural issues and outcomes after transcatheter aortic valve implantation. Eur Heart J. 2014;35:2627-38.

15. Sinning JM, Werner N, Nickenig G, Grube E. Transcatheter aortic valve implantation: the evidence. Heart. 2012;98 Suppl 4:iv65-72.

16. Thomas M, Schymik G, Walther T, Himbert D, Lefèvre T, Treede H, et al. Thirty-day results of the SAPIEN aortic Bioprosthesis European Outcome (SOURCE) Registry: a European registry of transcatheter aortic valve implantation using the Edwards SAPIEN valve. Circulation. 2010;122:62-9.

17. Moat NE, Ludman P, De Belder MA, Bridgewater B, Cunningham AD, Young CP, et al. Long-term outcomes after transcatheter aortic valve implantation in high-risk patients with severe aortic stenosis: the UK TAVI (United Kingdom Transcatheter Aortic Valve Implantation) Registry. J Am Coll Cardiol. 2011;58:2130-8.

18. Gilard M, Eltchaninoff $H$, lung $B$, Donzeau-Gouge P, Chevreul K, Fajadet J, et al. FRANCE 2 Registry of transcatheter aortic-valve implantation in high-risk patients. N Engl J Med. 2012;366:1705-15.

19. Zahn R, Gerckens U, Grube E, Linke A, Sievert H, Eggebrecht H, et al. Transcatheter aortic valve implantation: first results from a multi-centre real-world registry. Eur Heart J. 2011;32:198 204

20. Hamm CW, Möllmann H, Holzhey D, Beckmann A, Veit C, Figulla HR, et al. The German Aortic Valve Registry (GARY): in-hospital outcome. Eur Heart J. 2014;35:158898.

21. Mohr FW, Holzhey D, Möllmann H, Beckmann A, Veit C, Figulla HR, et al. The German Aortic Valve Registry: 1-year results from 13680 patients with aortic valve disease. Eur J Cardiothorac Surg. 2014;46:808-16.

22. Tamburino C, Capodanno D, Ramondo A, Petronio AS, Ettori F, Santoro G, et al. Incidence and predictors of early and late mortality after transcatheter aortic valve implantation in 663 patients with severe aortic stenosis. Circulation. 2011;123:23941.

23. Ussia GP, Barbanti M, Petronio AS, Tarantini G, Ettori F, Colombo A, et al. Transcatheter aortic valve implantation: 3-year outcomes of self-expanding CoreValve prosthesis. Eur Heart J. 2012;33:969-76.

24. Muñoz-García AJ, Del Valle R, Trillo-Nouche R, Elízaga J, Gimeno F, Hernández-Antolín $R$, et al. The Ibero-American transcatheter aortic valve implantationregistry with the CoreValve p rosthesis. Early and long-term results. Int J Cardiol. 2013;169:35965.

25. Avanzas P, Muñoz-García AJ, Segura J, Pan M, Alonso-Briales JH, Lozano I, et al. Implante percutáneo de la prótesis valvular aórtica autoexpandible CoreValve® en pacientes con estenosis aórtica severa: experiencia inicial en España. Rev Esp Cardiol. 2010;63:141-8.

26. Registro SOURCE. Disponible en: http://clinicaltrials.gov/show/NCT01238497

27. Linke A, Wenaweser P, Gerckens U, Tamburino C, Bosmans J, Bleiziffer S, et al. Treatment of aortic stenosis with a self-expanding transcatheter valve: the International Multi-centre ADVANCE Study. Eur Heart J. 2014;35:2672-84.

28. Toggweiler S, Humphries KH, Lee M, Binder RK, Moss RR, Freeman M, et al. 5-year outcome after transcatheter aortic valve implantation. J Am Coll Cardiol. 2013;61:413-9.

29. Roy DA, Schaefer U, Guetta V, Hildick-Smith D, Möllmann H, Dumonteil N, et al.Transcatheter aortic valve implantation for pure severe native aortic valve regurgitation. J Am Coll Cardiol. 2013;61:1577-84.

30. Dvir D, Webb J, Brecker S, Bleiziffer S, Hildick-Smith D, Colombo A, et al. Transcatheter aortic valve replacement for degenerative bioprosthetic surgical valves: results from the global valve-in-valve registry. Circulation. 2012;126:2335-44.

31. Ribeiro HB, Webb JG, Makkar RR, Cohen MG, Kapadia SR, Kodali S, et al. Predictive factors, management and clinical outcomes of coronary obstruction following transcatheter aortic valve implantation: insights from a large multicenter registry. J Am Coll Cardiol. 2013;62:1552-62.

32. Yousef A, Simard T, Pourdjabbar A, Webb J, So D, Chong AY, et al. Performance of transcatheter aortic valve implantation in patients with bicuspid aortic valve: Systematic review. Int J Cardiol. 2014;176:562-4. 